\title{
Habitable Exoplanet Observatory (HabEx) telescope and optical instruments
}

Stefan Martin, Gary Kuan, Daniel Stern, Paul Scowen, John Krist, et al.

Stefan Martin, Gary Kuan, Daniel Stern, Paul Scowen, John Krist, Dimitri Mawet, Garreth Ruane, "Habitable Exoplanet Observatory (HabEx) telescope and optical instruments," Proc. SPIE 11117, Techniques and Instrumentation for Detection of Exoplanets IX, 1111704 (9 September 2019); doi: 10.1117/12.2530737

EDent: SPIE Optical Engineering + Applications, 2019, San Diego, California, United States 


\title{
Habitable Exoplanet Observatory (HabEx) Telescope and Optical Instruments.
}

\author{
Stefan Martin*, Gary Kuan*, Daniel Stern*, \\ Paul Scowen**, John Krist*, Dimitri Mawet***, Garreth Ruane*, \\ *Jet Propulsion Laboratory, California Institute of Technology, Pasadena, CA 91109. \\ **Arizona State University, Tempe, AZ 85287, \\ ***Caltech, 1200 E. California Blvd., Pasadena, CA 91125
}

\begin{abstract}
The HabEx study has developed a baseline concept for a $4 \mathrm{~m}$ aperture next generation space telescope operating from the ultraviolet to the infrared, capable of compelling general astrophysics and exoplanet science. HabEx carries four instruments, a UV spectrometer/imager (UVS) together with a general purpose astrophysics camera/spectrograph (HWC) and for exoplanet work, a coronagraph and a starshade. UVS reaches down to $115 \mathrm{~nm}$ with resolution up to 60,000 and a 3'x3' field of view. HWC operates between $370 \mathrm{~nm}$ and $1800 \mathrm{~nm}$, again with a 3'x3' field of view; the spectral resolution is 1000 and it carries a suite of science filters. The telescope is capable of tracking both deep space and solar system objects. The coronagraph enables observations and spectroscopy at up to $\mathrm{R}=140$ with instantaneous $20 \%$ bandwidth between wavelengths of 450 and $1800 \mathrm{~nm}$ and is intended to be used in a survey mode. However, it also backs up most of the functionality of the accompanying starshade instrument which will have superior performance for spectroscopy. The $52 \mathrm{~m}$ diameter starshade flies 76,600 $\mathrm{km}$ from the telescope and at that range has a broadband suppression of the starlight between 300 and $1000 \mathrm{~nm}$. A single observation at $108 \%$ bandwidth covers a very wide spectral band at a resolution up to 140. Both coronagraph and starshade are equipped with integral field spectrometers to enable simultaneous spectroscopy of exoplanets within the field of view. This paper details the design of the telescope, the four science instruments and associated optical systems.
\end{abstract}

Keywords: HabEx, Exoplanet, Starshade, Coronagraphy, Ultraviolet, Infrared, Spectroscopy, Imaging.

\section{INTRODUCTION}

The baseline HabEx mission is composed of two separate spacecraft flying in formation in an Earth-Sun L2 orbit. One spacecraft carries a $4 \mathrm{~m}$ off-axis telescope and four science instruments: a coronagraph (CG) and a starshade instrument (SSI) for exoplanet direct imaging, and two wide field instruments, the workhorse camera (HWC) and the highresolution ultraviolet spectrograph (UVS) for observatory science. The second spacecraft carries a $52 \mathrm{~m}$ starshade. Together they form an external occulter observatory for exoplanet imaging and spectral characterization. The starshade suppresses the light from the target star while the telescope's starshade instrument observes the planetary system surrounding the target star. To form the observatory, the starshade is positioned into the line of sight (LOS) between the telescope and the target star at a 76,600 km separation from the telescope, and maintains alignment using a positional control loop carried over a spacecraft-to-spacecraft radio link. Longer and shorter starshade ranges will also be used depending on the observing band. Starshade lateral and longitudinal position sensing are carried out by optical instruments on the telescope spacecraft and position control is handled by the propulsion system on the starshade spacecraft.

The telescope spacecraft launches on a SLS Block 1B launch vehicle into an L2 orbit. The starshade launches separately on a Falcon Heavy. The primary mission will run for five years, but the telescope includes enough fuel to continue operations for at least five additional years. The starshade spacecraft has fuel for five years of operations after which it can no longer transit to new targets and must hold the L2 orbit until serviced. Indeed, both spacecraft are serviceable and can be refueled and in addition the telescope can be upgraded with new science instruments.

The coronagraph and starshade instruments are complementary in nature. While the starshade instrument (SSI) is capable of very high-contrast imaging and spectroscopy over a large field of view (FOV), it is limited in the number of observations due to the large slew times of the starshade. The coronagraph, on the other hand, is capable of faster slews, making many more observations possible, but has a narrower, high-contrast FOV with reduced spectrographic

Techniques and Instrumentation for Detection of Exoplanets IX, edited by Stuart B. Shaklan, Proc. of SPIE Vol. 11117, 1111704 - (c) 2019 SPIE · CCC code: 0277-786X/19/\$21 - doi: 10.1117/12.2530737 
capability. Working together, the coronagraph can be used in a survey mode to determine orbits, and the starshade would be used in a follow-up mode to provide the spectroscopic data needed to characterize exoplanet atmospheric gases. In addition, the inclusion of imaging and spectroscopy capabilities within both instruments adds resiliency against technical and programmatic risks; loss of one exoplanet instrument does not eliminate the exoplanet science return of the mission since both the coronagraph and starshade instruments carry imaging and spectroscopy channels with similar (but necessarily not identical) capabilities.

HabEx employs an off-axis, unobscured monolithic telescope architecture to maximize coronagraph efficiency while minimizing telescope complexity. Maintenance of the coronagraph contrast performance requires that the Optical Telescope Assembly (OTA) be ultra-stable in both internal optical alignment and optical surface figure. Laser metrology and control (MET) maintains the alignment of the secondary mirror (SM) and tertiary mirror assembly (TMA) with respect to the primary mirror (PM). The telescope also includes precision thermal control and a fine guidance sensor system (FGS) paired with an attitude control system employing microthrusters for pointing control during observations. The use of microthrusters rather than reaction wheels avoids the introduction of high frequency jitter. Microthrusters in fact present an almost ideal solution for accurate attitude control with a coronagraph, which is acutely sensitive to any wavefront fluctuations. Microthrusters produce less than $0.1 \mu \mathrm{N} / \sqrt{\mathrm{Hz}}$ noise PSD up to $1 \mathrm{~Hz}$, orders of magnitude less than typical reaction wheels and eliminating the need for vibration isolation between the payload and spacecraft.

\subsection{Bus Payload}

The baseline optical telescope assembly ${ }^{[1]}$ (OTA, Figure 1) consists of the primary and tertiary mirror (PM \& TM) assemblies, secondary mirror (SM) assembly, secondary mirror tower with integrated science instrument module, and stray-light tube with forward scarf. The scarf angle of $45^{\circ}$ determines the closest angle of observation to the sun. The tower and baffle tube form an optical bench which maintains alignment between the PM, SM and TM assemblies. The solar arrays located on the side and base of the spacecraft are sized to meet thermal power requirements during anti-Sun pointing. Conventional thrusters are used for slewing the observatory and microthrusters are used for fine pointing control during science observations. The OTA and spacecraft connect only at the interface ring which is also the interface between the payload and the launch vehicle payload adapter fixture (PAF) connecting to the Space Launch System (SLS). A telescope forward door is closed for launch to prevent contamination and also provides additional initial stiffness.

The spacecraft surrounds the telescope without physically touching it except at the interface ring minimizing the propagation of mechanical disturbances from the spacecraft into the telescope and providing good thermal isolation of the telescope. While the primary and secondary mirrors have active thermal control, the structure does not because the MET system maintains alignment between the primary, secondary and tertiary mirrors. The MET system senses and corrects slow thermal drifts and its noise uncertainty is easily sufficient to meet the WFE stability requirements.

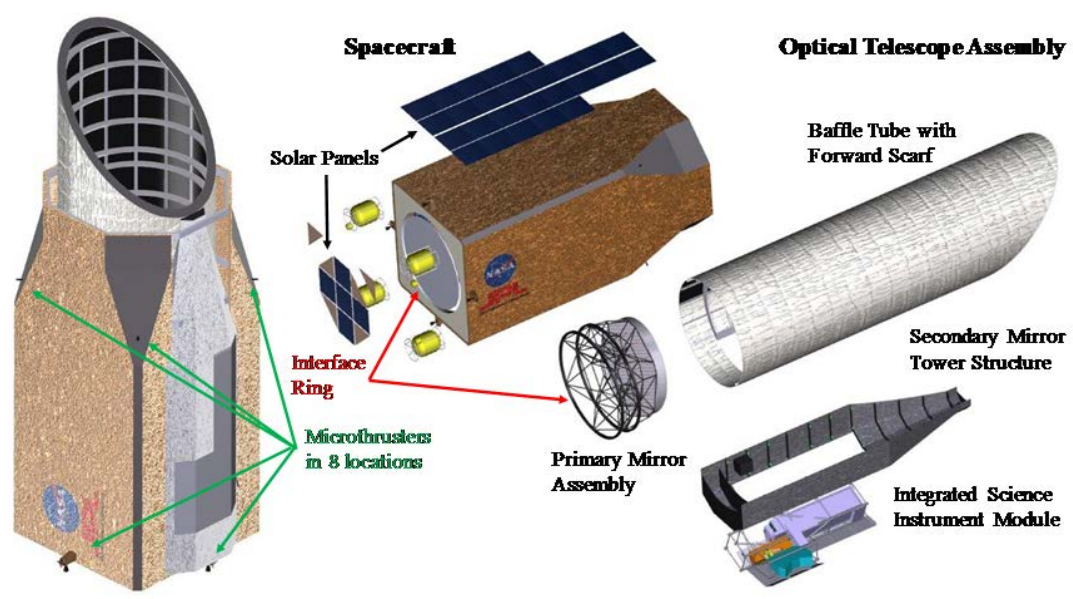

Figure 1: HabEx telescope components, showing the assembled telescope and the principal components. 


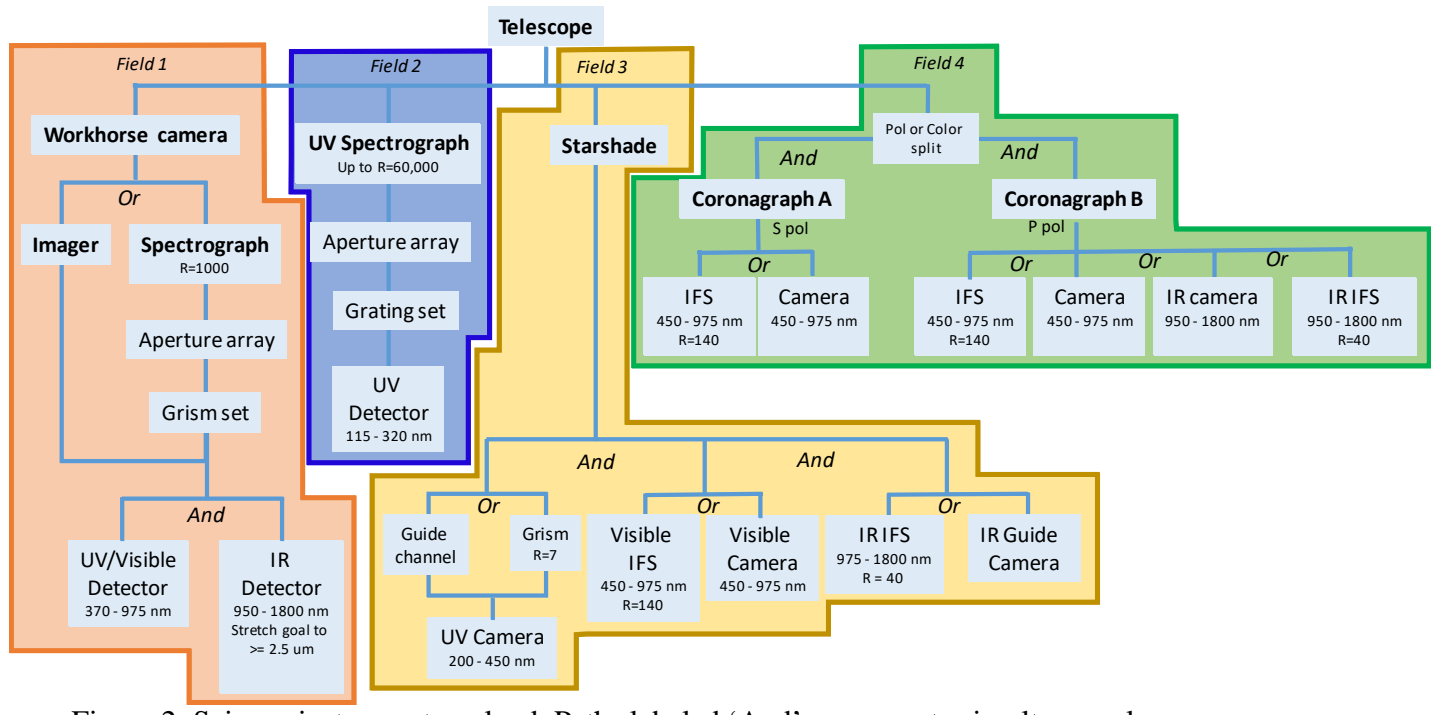

Figure 2: Science instrument payload. Paths labeled ‘And’ can operate simultaneously.

\section{INSTRUMENTS AND TELESCOPE}

The telescope's scientific payload ${ }^{[2]}$ consists of the four science instruments (Figure 2), plus ancillary equipment. The coronagraph can make rapid survey observations while the starshade camera can observe angles closer to the star and once on target, enables more efficient spectroscopic observations. The coronagraph (CG) contains two channels, one for each orthogonal input polarization, capable of covering a broad spectral range from $450 \mathrm{~nm}$ to $1000 \mathrm{~nm}$ in four observations. The coronagraph channels can also be configured to operate with a color/polarization split so that one operates on "blue" light and the other on orthogonally polarized "red", for example. Also, the coronagraph has an IR channel operating up to $1800 \mathrm{~nm}$.

The starshade instrument (SSI), in conjunction with the external starshade covers the wavelength range $300 \mathrm{~nm}$ to 1000
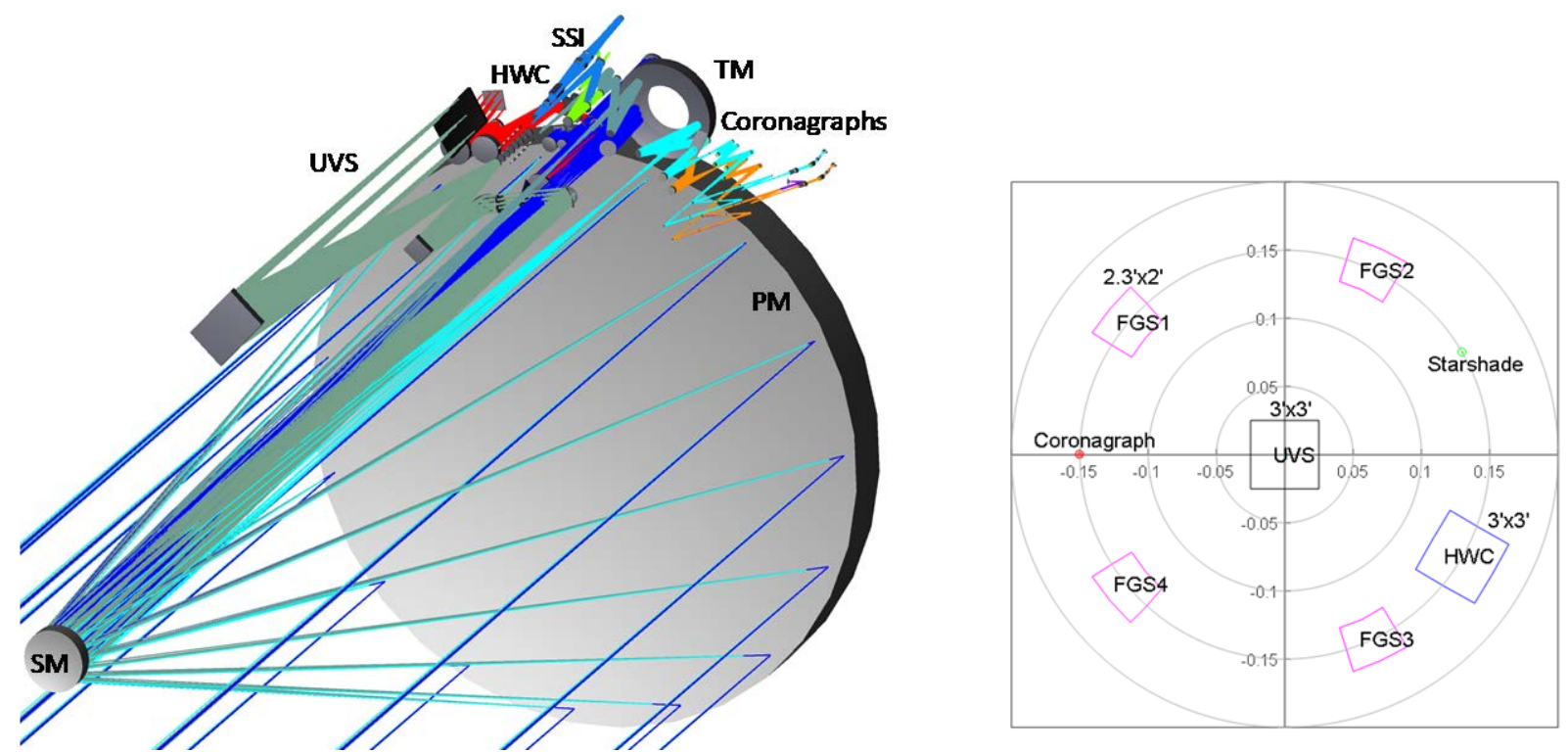

Figure 3: Left, telescope optical layout showing the science instruments alongside the telescope barrel. Right, fields of view on the sky. 
$\mathrm{nm}$ in one observation. It also offers observation capability into the infrared up to $1800 \mathrm{~nm}$ and down into the ultraviolet to $200 \mathrm{~nm}$.

The first observatory general astrophysics science instrument is a high-resolution ultraviolet spectrograph and camera (UVS) operating from $115 \mathrm{~nm}$ to $320 \mathrm{~nm}$ with resolution R up to 60,000 on a 3' $\times 3^{\prime}$ field of view. The second observatory science instrument is also a camera and spectrograph (HWC) enabling imaging and spectroscopy, also on a $3^{\prime} \times 3^{\prime}$ field of view, in two bands stretching from the UV to the near-IR. In spectroscopy mode, the HWC operates as a multi-object spectrograph with resolution of 1,000 .

The ancillary optical payload equipment consists of the laser metrology system (MET) and a fine guidance sensor system (FGS) to maintain the pointing stability required for efficient observations.

\subsection{Optical Telescope}

The HabEx telescope is an unobscured three mirror anastigmat design (TMA) with a $4 \mathrm{~m}$ diameter primary mirror, f\# 2.5, $2.5 \mathrm{~m}$ off axis, producing a collimated $50 \mathrm{~mm}$ beam at the output. The TMA design allows the minimization of the three principal optical aberrations (spherical, coma, astigmatism) and furthermore, field curvature can be brought to zero by choosing appropriate mirror figures. Such a design provides an extremely well corrected wavefront over a wide field of view, allowing the science instruments to operate simultaneously in different areas of the sky. Figure 3 shows the telescope's optical layout. A 4 m primary mirror (M1) directs light to the secondary (M2) then onto the tertiary (M3). The relatively long f/number of 2.5, was chosen to control polarization mixing for high contrast coronagraphy.

Since the telescope supports UV observations, a protected aluminum coating is required on at least the first two mirrors and sensitivity to mirror contamination in the UV part of the spectrum is a factor setting the operating temperature for the primary mirror: too low a temperature would result in the condensation of contaminants on the mirror surfaces, drastically reducing UV throughput. The instruments are arranged on the side of the telescope near M3. Compared to mounting instruments below the primary mirror, the side mounting allows easier extraction of the instrument modules for servicing and takes advantage of volume created by the presence of the tertiary mirror beside the primary. Furthermore, it creates easy access to externally mounted radiators needed for cooling the detectors. Rear mounting would make use of space behind the primary, between its supporting structure and the bus, but may increase the overall telescope height (already greater than $10 \mathrm{~m}$ ). Cooling paths would generally be longer and extraction of individual modules might be more complex. However, both design concepts are viable and would occupy similar volumes.

Figure 3 left shows the instruments in position near the primary mirror. Rays from the secondary come from the lower left of the figure and the various instrument fields of view become separated at the tertiary mirror M3 (TM). Figure 3 right shows the on-sky fields of view of the instruments. The UVS occupies the center of the field and the coronagraph views a smaller field to the left. The starshade instrument views a region to the right and slightly upwards while the HWC views a wide area to the right and slightly downwards. Arranged around the rest of the tertiary mirror are the four fine guidance sensor areas.

After striking the tertiary, the rays are collimated and converge towards a common on axis pupil plane. Before reaching that plane, however, the beams are extracted by fold mirrors and directed to the instruments. An exception is the UVS, which has its own tertiary and the beam is extracted prior to the Cassegrain focus using a fold mirror.

\subsection{HabEx Coronagraph}

Collimated light from the telescope's M3 mirror enters the coronagraph instrument (Figure 4), with the first optic being a Fine Steering Mirror (FSM) placed at a pupil image plane. The FSM is used for pointing control. The collimated beam is then passed to a pair of deformable mirrors (DMs) for wavefront correction. Following the DMs, the beam is focused using a parabolic mirror onto a coronagraphic mask. The mask design has a coating that reflects out of band light to the Zernike Wavefront Sensor (ZWFS) ${ }^{[3]}$. The ZWFS is used in conjunction with the DMs to sense and correct tip and tilt. Light transmitted through the mask is recollimated with a second parabolic mirror and reaches the Lyot stop located at the exit pupil. The Lyot stop blocks the rejected starlight. Finally, the light enters either a camera or an integral field spectrograph $\left(\mathrm{IFS}^{[4]}\right)$ for imaging and spectral functions. Table 1 shows the functional design parameters. To provide the wavelength range, the coronagraph has both visible and IR detectors. 


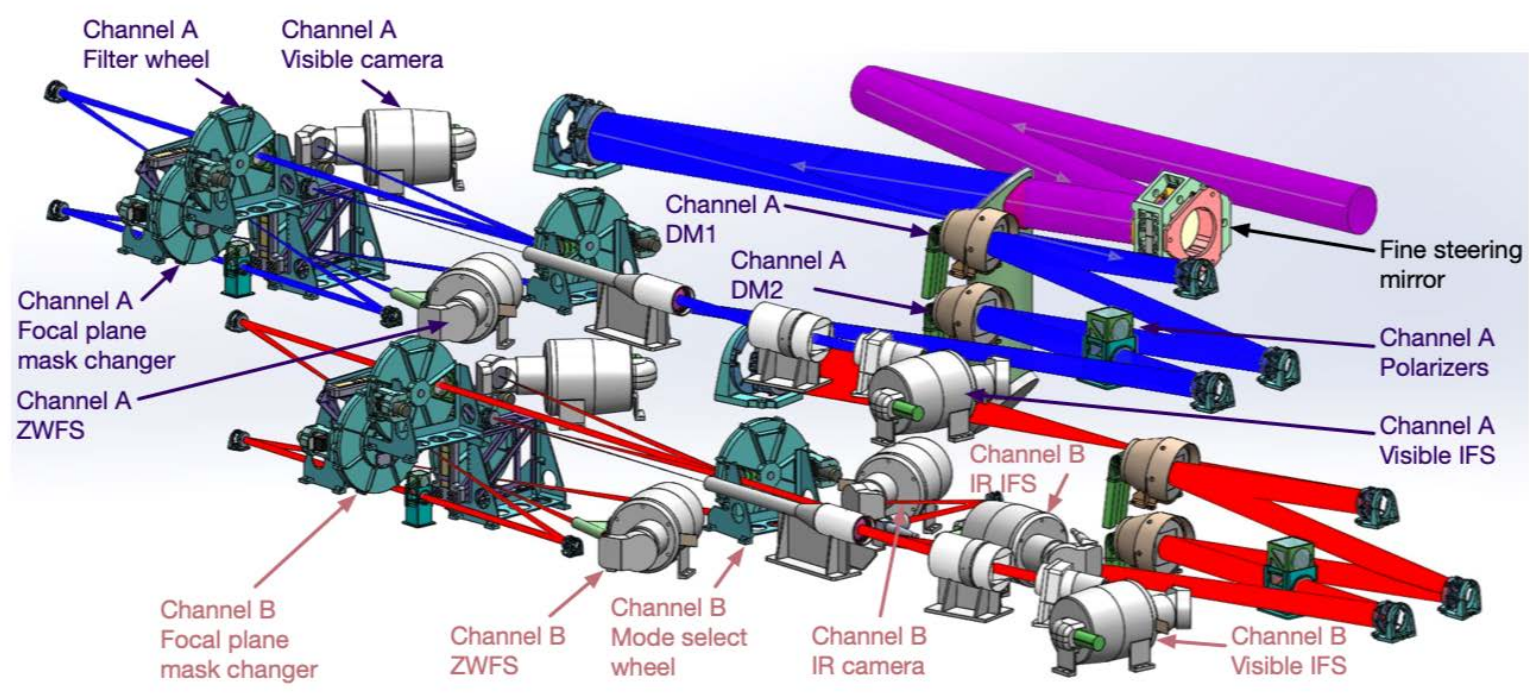

Figure 4: Coronagraph optomechanical layout showing A \& B channels. Cylindrical objects are camera housings and the various filter and mask wheels share a common design.

To observe efficiently, two near-identical coronagraphs are specified within the one coronagraph instrument, identified as Channel A and Channel B and observing the exact same field (Figure 3, right). Depending on the observation strategy, light entering the instrument is split into two bands. Under strategy one, the splitter is a polarization beam splitter and under strategy two it could either be a polarization beam splitter or a dichroic (also with polarizing function). Strategy one: a polarization beam splitter transmits vertically polarized light to Channel A and reflects horizontally polarized light to Channel B. Within the two channels, dichroic filters set the optical bandwidth to $20 \%$. Strategy two: a polarization beam splitter transmits vertically polarized light to Channel A and reflects horizontally polarized light to Channel B. Within the two channels, dichroic filters set the optical bandwidth to $20 \%$ utilizing one short wavelength

Table 1: Coronagraph design parameters: Channels A \& B operate in orthogonal polarizations and are identical except that B carries an IR capability.

\begin{tabular}{|c|c|c|}
\hline \multicolumn{3}{|c|}{ Camera Channels } \\
\hline & Visible Channel (A\&B) & IR Channel (B) \\
\hline FOV & $1.5^{\prime \prime}-2.7^{\prime \prime}$ & $3.1^{\prime \prime}$ \\
\hline Wavelength bands & $450-1000 \mathrm{~nm}$ (4 20\% bands) & $950-1800 \mathrm{~nm}$ (4 20\% bands) \\
\hline Pixel resolution & 11.6 mas- 17.3 mas & 29.9 mas \\
\hline IWA (2.4 N/D) & $56-102$ mas & 118 mas (at $0.95 \mu \mathrm{m}$ ) \\
\hline OWA (as) & $0.74-1.35$ & $1.57-1.90$ \\
\hline Detector & $1 \times 1$ CCD201 & $1 \times 1$ LMAPD \\
\hline Array width & 1024 & $256 \times 320$ \\
\hline \multicolumn{3}{|c|}{ Spectrograph Channels } \\
\hline & Visible Channel (A\&B) & IR Channel (B) \\
\hline FOV & $1.5^{\prime \prime}-2.7^{\prime \prime}$ & $3.1^{\prime \prime}$ \\
\hline Spectrometer resolution & 140 & 40 \\
\hline Spectrometer type & IFS & IFS \\
\hline Detector & 1/4 CCD282 (EMCCD) & $2 \times 2$ LMAPD* \\
\hline Array width (pixels) & 2048 & 2048 \\
\hline \multicolumn{3}{|c|}{ Other } \\
\hline Deformable mirror & \multicolumn{2}{|c|}{2 mirrors, 64 elements wide, $0.4 \mathrm{~mm}$ pitch } \\
\hline \multirow[t]{2}{*}{ Polarization } & Vertical (A channel) & Horizontal (primarily) \\
\hline & Horizontal (B channel) & Vertical (possible) \\
\hline
\end{tabular}


filter and longer wavelength filter top cover a broader band. Each coronagraph channel carries a camera and an IFS, selected by inserting a mirror. An infrared channel resides on coronagraph Channel B and is selected similarly. It carries an infrared IFS (a high resolution infrared fiber spectrograph would be an alternative) with $\mathrm{R}=40$ and covers the range 950-1800 $\mathrm{nm}$ in three 20\% wavelength bands. An infrared linear mode avalanche photodiode detector (LMAPD) is used on this channel.

Two deformable mirrors (DMs) allow the correction of both the wavefront phase and amplitude. For highest efficiency, the first DM would be placed at a pupil plane (correcting phase) and the second at the $1 / 4$ Talbot length away (correcting amplitude). Following modeling, the distance has been modified to $1 / 8$ Talbot length as it was found that a shorter distance improved the overall wavefront correction across the spectral band. Following the coronagraph mask, small wavefront imperfections arising from upstream optical elements and mask defects interfere to create a wavelengthdependent speckled light field at the focal plane. While a perfect correction of the wavefront error across the whole focal plane and across all wavelengths is not possible, the DMs are used to create a 'dark hole' over a restricted annular area of the focal plane. The coronagraph channels (depicted schematically in Figure 5) follow a similar layout to the WFIRST coronagraph design, while attempting to minimize the number of mirrors needed so as to maintain optical throughput. Following the common fine-steering mirror, the A and B channels are separated in collimated space by a selectable dichroic beam splitter. An initial relay of two off-axis parabolas (OAPs) sets the magnification to place the pupil on the DM. Telecentricity is not preserved at the entrance but is restored after the relay so that DM1 is positioned at a pupil plane. Following a fold, the beam strikes DM2 and is then focused onto the coronagraphic mask.

Light reflected by the mask is directed to a Zernike wavefront sensor (ZWFS). Following recollimation of the science beam, it is apertured at the Lyot stop and after the stop the light can be directed via selector mirrors to the IFS, to the camera, or in the case of the B channel, to the IR camera/spectrograph. A filter wheel allows selection of the appropriate wavelength bands. Polarizers are included in the beam train to allow operation with the vector vortex masks and also to allow selection of polarized light from the science targets, for example during disk imaging.

The optical throughput of the instrument is shown in Figure 6 left, excluding detection factors such as QE and "core” PSF (point spread function) throughput. Surface coatings are all protected silver for the mirrors, except M1 and M2, which are protected aluminum. At $700 \mathrm{~nm}$, the transmittance for the camera channel is $26 \%$, while the IFS channel is $\sim 15 \%$.

\subsection{Coronagraphic Masks}

The collimated beam reflecting off DM2 is brought to a focus by an off-axis parabolic mirror with a focal ratio of f/30. The focused star image has a PSF core Airy disc diameter of $42 \mu \mathrm{m}$. The coronagraphic mask element is placed at this focal plane. To cover the entire HabEx bands within both the visible and infrared channels, several masks are needed to provide the best starlight suppression. These masks are carried by a wheel mechanism, with the appropriate mask rotated into position depending on the science waveband selected.

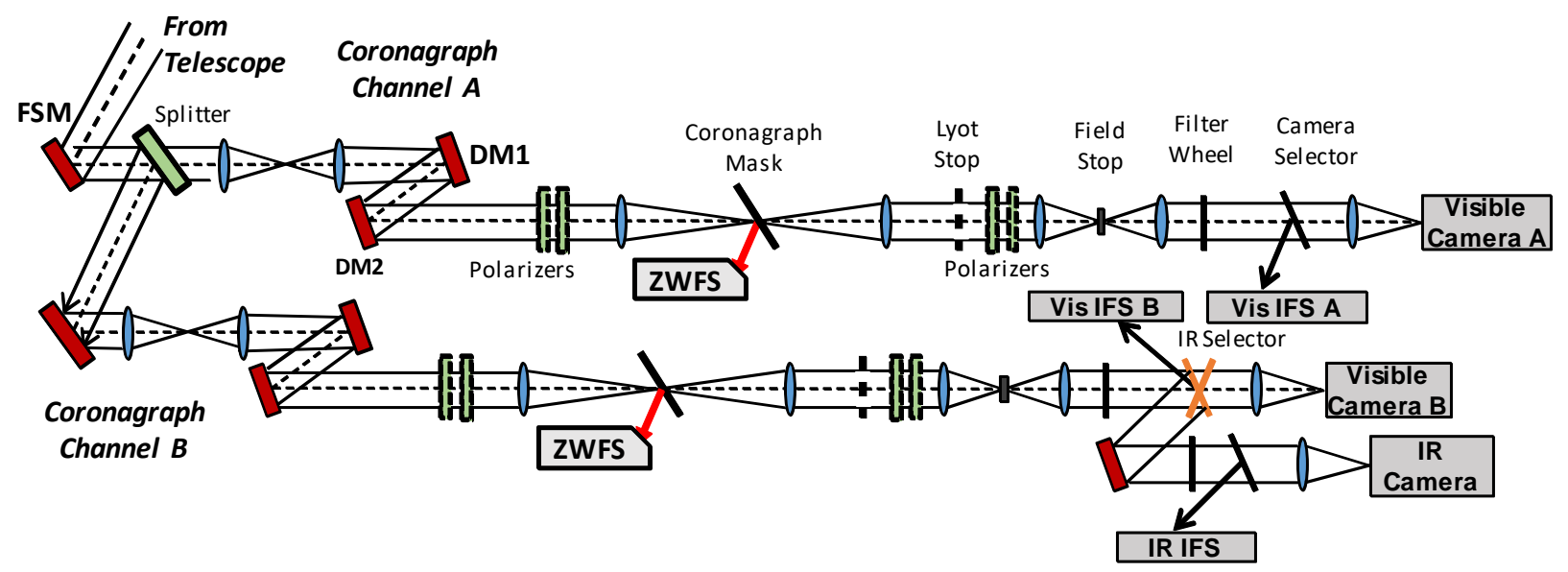

Figure 5: Two coronagraph channels provide visible through infrared operation. A \& B channels operate simultaneously, with the either the visible of IR sections of B selected towards the end of the beam train. 

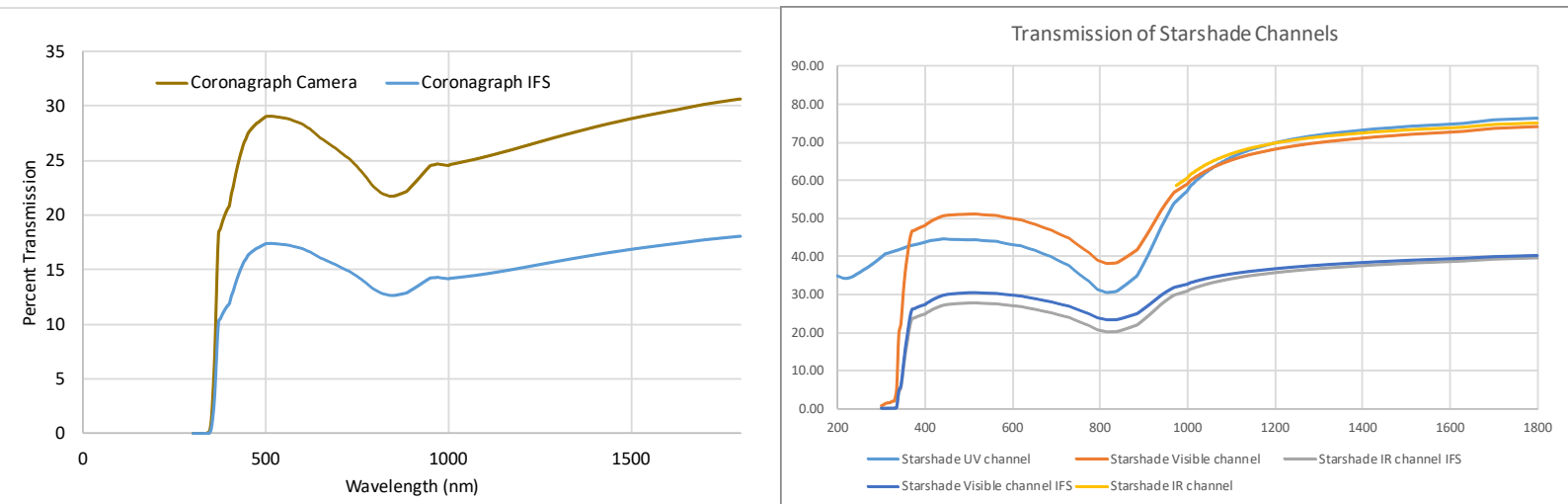

Figure 6: Transmission of coronagraph and starshade channels, showing $~ 60 \%$ more transmission for the starshade camera compared to the coronagraph.

The vortex coronagraph is a phase-mask coronagraph, requiring only a focal-plane mask and a standard circular Lyot stop. The vortex phase mask has a topological charge which refers to the number of phase wraps provided by the phase ramp. Higher topological charges produce less sensitivity to low order optical aberrations resulting in larger IWAs (inner working angles), so the choice of charge is an important factor. State of the art vortex coronagraph masks ${ }^{[5]}$ have been built using one of the following technologies: liquid crystal polymers (LCP), photonics crystals (PC), or subwavelength gratings. The best lab results for vortex masks have been obtained on the High Contrast Imaging Testbed (HCIT) with the LCP approach.

For Habex, a vortex mask of topological charge 6 represents (for the majority of science targets) the optimal trade-off between inner working angle (2.4 $\lambda / \mathrm{D}$ at $50 \%$ total off-axis throughput: $\mathrm{IWA}_{0.5}$ ), and immunity to low-order aberrations (tip-tilt, defocus, astigmatism, coma, spherical). No other mask architecture so far found matches the charge 6 vortex's performance in this regard. The Hybrid Lyot Coronagraph (HLC) mask has high technology readiness (TRL 5) and traceability to the WFIRST CGI, but is considered only a potential backup due to its poor immunity to low order wavefront errors (the coronagraph design can also accept HLC masks).

\subsection{Zernike Wavefront Sensor (ZWFS)}

The VVC mask is slightly tilted with respect to the beam path and has a dichroic surface that sends a portion of the starlight into the ZWFS. Initially, WFE introduced by the optical system is corrected by the two DMs which are adjusted to produce a very dark annular hole across the $20 \%$ bandwidth, with some residual speckles. During observations, which may take many hours, the wavefront slowly evolves under small thermal changes producing changes in the speckles on the coronagraph focal plane. Since these speckles limit the ability to detect exoplanets they must be controlled. Changes to tip/tilt and focus will be detected at the ZWFS. This sensor allows detection of tip/tilt and focus errors at submilliarcsecond levels, so that tip/tilt can be corrected by the FSM at the entrance to the coronagraph. Once the spacecraft has been slewed to a target (or reference) star and stabilized, an acquisition process results in the star being centered on the coronagraph's occulting mask, with starlight outside the science band reflecting off the mask. This light is re-imaged by optical elements onto the ZWFS detector, creating a pseudo-interferogram. Motion of the telescope creates a change in the interferogram and the resulting error signal is fed back to the FSM to correct.

The ZWFS sensor is similar to the WFIRST CGI's LOWFS. The ZWFS is based on the Zernike phase contrasting principle where a small $(\sim 1 \lambda / \mathrm{D})$ diameter phase dimple with phase difference of $\sim \pi / 2$ is placed at center of the starlight PSF. The phase-modulated PSF light is then collimated and forms a pupil image at the ZWFS camera. The interference between the light that passes inside and outside the phase dimple converts the wavefront phase error into the measurable intensity variations in the pupil image on the ZWFS camera. The spatial sampling of the pupil image on the ZWFS camera depends on the spatial frequency of the wavefront errors to be sensed. On WFIRST CGI, LOWFS's camera is running at high frequency ( $1 \mathrm{kHz}$ frame rate) in order to sense the fast LOS jitter from the vibration sources such as telescope's reaction wheels, and correct it using the fast steering mirror at the coronagraph entrance. For HabEx, this high frame rate is not required because of the lack of high frequency disturbance from the microthrusters. A control loop operates on the FSM to correct the telescope's LOS to a level higher ( $\leq 0.2$ mas per axis) than that achieved by the FGS (0.7 mas per axis). The telescope stability is such that higher order ZWFS-sensed wavefront error modes need not be 
Table 2: Starshade instrument design parameters. The operating parameters of the camera channels are shown, as distinct from the wider starshade bands. Thus, two cameras would be used to cover the each range-dependent starshade science band.

\begin{tabular}{|c|c|c|c|}
\hline Cameras & $\begin{array}{c}\text { UV } \\
\text { Channel }\end{array}$ & $\begin{array}{l}\text { Visible } \\
\text { Channel }\end{array}$ & $\begin{array}{l}\text { IR Guide } \\
\text { Channel }\end{array}$ \\
\hline FOV & $10^{\prime \prime}$ & $12^{\prime \prime}$ & 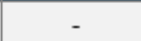 \\
\hline $\begin{array}{l}\text { Wavelength bands } \\
\text { (nm) }\end{array}$ & $200-450$ & $450-975$ & $975-1800$ \\
\hline Pixel resolution & 14.2 mas & 14.2 mas & $12 \mathrm{~cm}$ \\
\hline Telescope resolution & 21 mas & 21 mas & - \\
\hline Detector & $\begin{array}{c}1 \times 1 \\
\text { CCD201 }\end{array}$ & $\begin{array}{c}1 \times 1 \\
\text { CCD201 }\end{array}$ & 1×1 LMAPD \\
\hline Array width (pixels) & 1024 & 1024 & 256 \\
\hline Spe & $\begin{array}{c}\text { UV } \\
\text { Channel }\end{array}$ & $\begin{array}{l}\text { Visible } \\
\text { Channel }\end{array}$ & $\begin{array}{c}\text { IR } \\
\text { Channel }\end{array}$ \\
\hline FOV & $10^{\prime \prime}$ & $2^{\prime \prime}$ & $4^{\prime \prime}$ \\
\hline $\begin{array}{l}\text { Wavelength bands } \\
(\mathrm{nm})\end{array}$ & $200-450$ & $450-975$ & $975-1800$ \\
\hline $\begin{array}{l}\text { Spectrometer } \\
\text { resolution }\end{array}$ & 7 & 140 & 40 \\
\hline Spectrometer type & Slit/grism & IFS & IFS \\
\hline Detector & $\begin{array}{c}1 \times 1 \\
\operatorname{CCD} 201\end{array}$ & $\begin{array}{c}1 \times 1 \\
\text { CCD282 }\end{array}$ & 2×2 LMAPD \\
\hline Array width (pixels & 1024 & 4,096 & 2,048 \\
\hline
\end{tabular}

corrected as they are extremely small. While in the first place, STOP modeling shows that sensing of these higher order modes is not required, modeling of the ZWFS shows that such measurements are possible on stars up to 9th magnitude at least, which could provide valuable telescope diagnostic information.

\subsection{Focal Planes}

Detector arrays for the visible channels are EMCCD types, selected because of their exceptionally low effective read noise. The imaging focal planes (blue and red channels) consist of a single EMCCD per channel operated at $153 \mathrm{~K}$. The chosen type is a modified CCD201 with delta doping and a thickened substrate together with a broadband "astro" coating giving response out to $1.0 \mu \mathrm{m}$. The pixel scale is as shown in Table 1 . The corresponding IFS focal planes consist of a modified, cut down version of CCD282, which is a $4 \mathrm{k} \times 4 \mathrm{k}$ device with a $2 \mathrm{k} \times 4 \mathrm{k}$ frame store at each end. The device format allows it to be cut in half along both axes forming a $2 \mathrm{k} \times 2 \mathrm{k}$ sensor area with a $2 \mathrm{k} \times 2 \mathrm{k}$ frame store. The device would be operated at $163 \mathrm{~K}$ to minimize dark current.

For the infrared, an HgCdTe avalanche photodiode array (Selex LMAPD) is baselined. The detector is cooled to 60K to minimize dark current. It has a low read noise with avalanche gains of 50 or more available.

\section{HABEX STARSHADE INSTRUMENT}

The starshade system consists of the starshade itself (attached to its spacecraft) and the starshade instrument. The needs of the STM result in a broadband starshade design accommodating the spectral range with three optical channels accommodating the UV, visible and infrared. Table 2 summarizes the principal instrument specifications. To provide the wavelength range, the starshade instrument has UV, visible and IR detectors and to provide the imaging and spectral functions, both cameras and spectrographs as shown in 2.

The HabEx starshade is of the numerically optimized type ${ }^{[6]}$ rather than hyper-Gaussian, producing a designed highsuppression wavelength band. Light of both shorter and longer wavelengths is attenuated but leaks into the shadow region and is used for starshade positioning. The basic parameters for the starshade are: diameter $52 \mathrm{~m}$ with twenty six $16 \mathrm{~m}$ long petals with a $20 \mathrm{~m}$ diameter inner disc. Figure 7 shows the starshade transmission functions for the three planned science bands, $200-670 \mathrm{~nm}, 300-1000 \mathrm{~nm}$, and $540 \mathrm{~nm}$ to $1800 \mathrm{~nm}$. When performing science at longer "red" wavelengths, shorter wavelength "blue" light is used for guiding and vice versa. In normal operation, the starshade operates in the band from 300 to $1000 \mathrm{~nm}$. To work in the UV, the starshade can be moved further away from the 


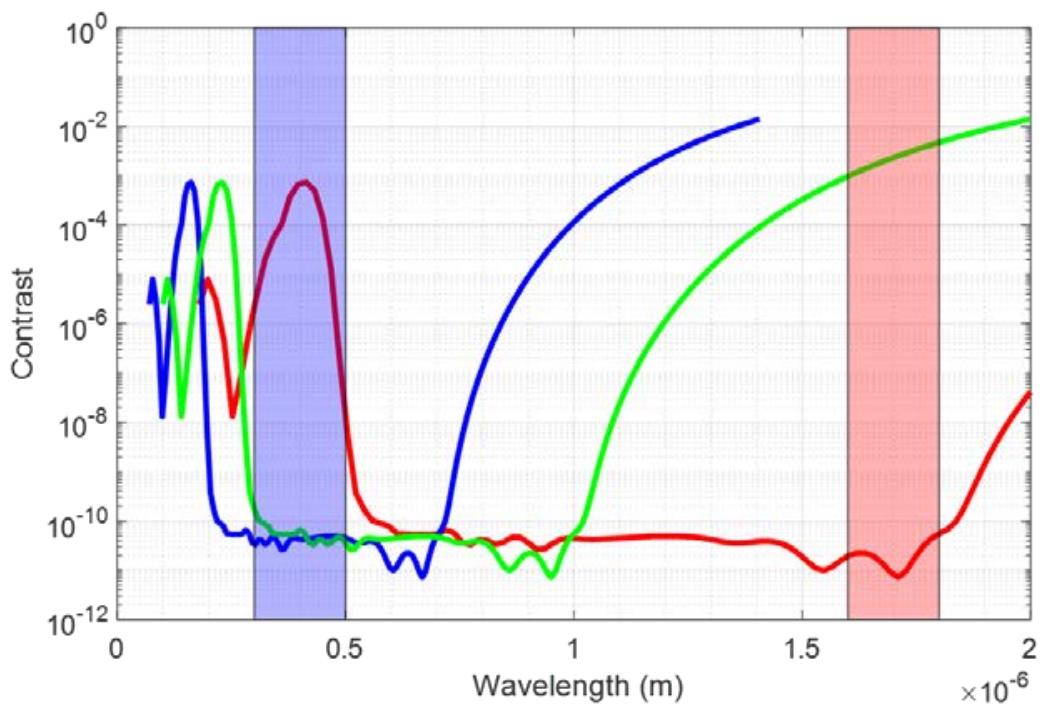

Figure 7: Starshade transmission function. Blue, green and red lines show the UV, visible and IR transmission bands. The rectangles indicate the guide channel bands with the red region being used for UV and visible science, and the blue for IR science.

telescope, achieving an IWA $\mathrm{A}_{0.5}$ of 47 mas. For infrared science, the starshade moves closer to the telescope and the IWA will increase in proportion to the wavelength. In the main 'visible' science band the starshade range is 76,610 m, with $\mathrm{IWA}_{0.5}$ of 58 mas (the $50 \%$ planet throughput point) and $\mathrm{IWA}_{\text {tip }}$ (at the tips of the petals) of 70 mas.

Starshade operation requires both science and formation flying cameras to view the starshade simultaneously. The telescope is placed as far back as possible within the high suppression (dark) shadow region that exists behind the starshade while maintaining high starlight suppression. The starshade can move laterally $\pm 1 \mathrm{~m}$ across the line of sight during observations.

\subsection{Design}

The starshade instrument contains six beam paths (Figure 8 shows an optical view) to accommodate three optical channels: UV, visible, and infrared. Light entering the starshade camera is split by dichroic optics into UV, then visible, and IR beam paths, so all of these channels can be operated simultaneously as seen in Figure 2. Camera and spectrograph properties are shown in Table 2. The UV channel carries a simple slit spectrograph employing a grism with $\mathrm{R}=7$. The visible channel carries a broadband IFS capable of covering the wavelength range from 450 to $975 \mathrm{~nm}$, plus an imaging camera for more rapid and wide-field system imaging. The infrared channel carries an IFS with R $=40$ to enable disc and object spectroscopy. The operation of these channels is detailed below.

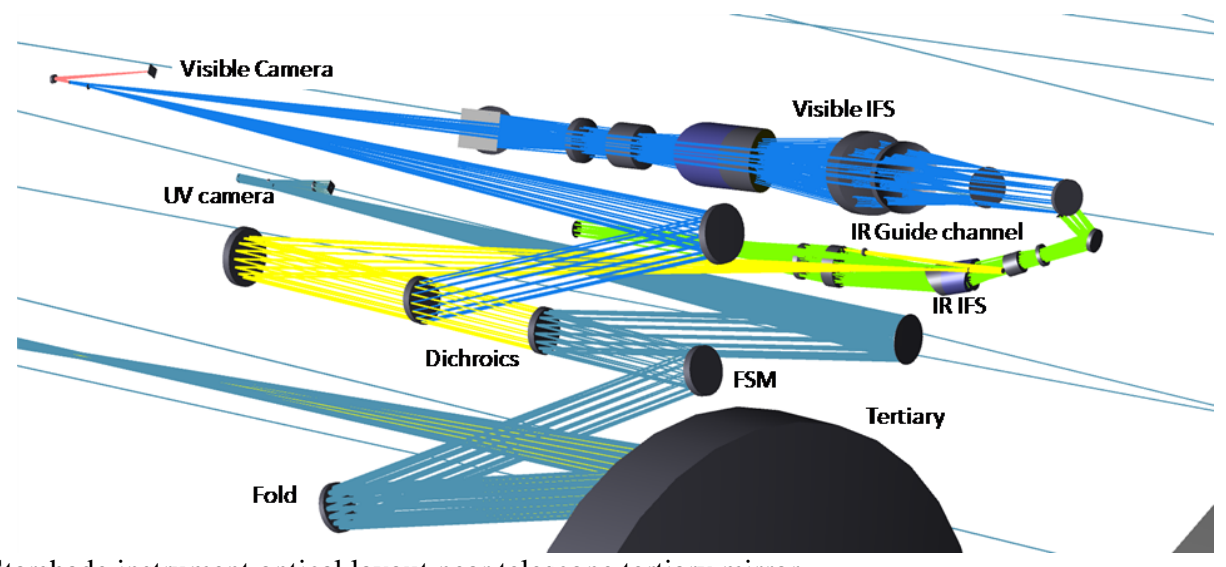

Figure 8: Starshade instrument optical layout near telescope tertiary mirror. 
The starshade's wide bandwidth provides high observational efficiency, and pairs of optical channels are employed to collect the light. When set up for 'green' science (referring to Figure 7), most of the science band is covered by the visible channel, but below $450 \mathrm{~nm}$, the UV channel covers the remainder down to $300 \mathrm{~nm}$. Similarly, for 'blue' science, the science band is covered by the visible and UV channels, and finally 'red' science is covered by the IR channel together with the visible channel. This sharing between the channels is expressed in the table: each column refers to a science mode, UV, Visible or IR, (corresponding to 'blue', 'green' and 'red' in Figure 7) and each row refers to a channel within the starshade instrument.

\subsection{Visible Channel}

The visible channel is the principal science channel and carries a camera and an IFS. The layout is shown schematically in Figure 9 center. Light from the telescope M3 strikes the fold mirror and then the FSM at the entrance to the starshade instrument. It then passes through a dichroic optic which reflects UV light. The remaining visible and infrared light passes to a second dichroic where the visible light is reflected to an off-axis paraboloidal (OAP) mirror and thence to a focus where field stops are inserted to limit the field of view, one for the imaging mode (11.9" diameter) and a second for spectroscopy (1.9" diameter). This focus is reimaged by an ellipsoidal mirror to the focal plane. A filter wheel is inserted after the ellipse with filters to select wavebands appropriate for different starshade ranges. For example, with the starshade at the nominal distance for visible work, the filter would pass 450 to $1000 \mathrm{~nm}$ light. With the starshade more distant as set up for UV science, the spectral range would be 450 to $670 \mathrm{~nm}$, beyond which point the starshade would start to leak redder light. Further filters and polarizing optics for polarization studies could also be inserted here, such as the science filters provided for coronagraphy.

The imaging focal plane consists of a single EMCCD operated at $153 \mathrm{~K}$. The chosen type is a modified CCD201 with delta doping and a thickened substrate together with a broadband "astro" coating giving response out to $1000 \mathrm{~nm}$ (Nikzad et al. 2017). The pixel scale is 14 mas. During a thruster firing, the sensor is read out at $1 \mathrm{kHz}$ to keep the accumulated photon count appreciably below full well.

For spectroscopy, an additional ellipsoidal mirror is inserted into the beam following the first ellipse, producing a large increase in the f/number from 47 to 1330. Via a fold mirror, this beam is focused onto a microlens array (MLA), which forms the entrance to the IFS. The IFS consists of the MLA, a matching multiple-aperture mask to restrict stray light, a set of lenses to collimate the beam, prisms to disperse the wavelengths and a second set of lenses to focus onto the focal plane. IFS operation can be visualized thus: for each microlens array element, one spectrum is produced on the focal plane. Since there is an array of microlenses, and array of spectra is produced and the optical system is designed to ensure that these spectra remain separate and distinct and do not overlap.

An image of the scene at one wavelength can be formed by using all the pixels on the focal plane that correspond to the same wavelength. A series of images known as "slices" can be assembled into a "data cube" with sides corresponding to the directions of the field of view and height corresponding to wavelength. Thus, the scene is reproduced in a stack of images representing narrow wavelength bands. To calibrate the images, it is necessary to provide a calibration source with at least one known wavelength in the band, and that illuminates the entire MLA. As shown in Figure 9 center, the calibration source light is injected when required through the fold mirror, which has a small leakage $2 \%$.

The IFS focal plane consists of a large single electron multiplying CCD (Teledyne/e2v CCD282) operated at $163 \mathrm{~K}$. The chosen type is a modified version of the off the shelf item with delta doping and a thickened substrate together with a
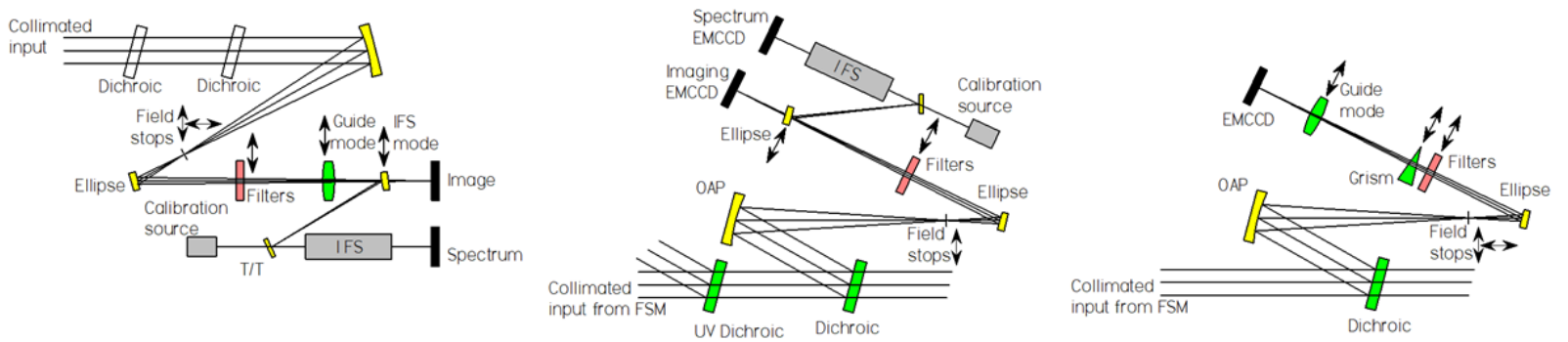

Figure 9: Layout of starshade instrument channels. Left to right, infrared, visible and UV channels. 
broadband "astro" coating giving response out to $1.0 \mu \mathrm{m}$. The format is an $8 \mathrm{k} \times 4 \mathrm{k}$ array with frame store areas at both sides of the $8 \mathrm{k}$ length, and a $4 \mathrm{k} \times 4 \mathrm{k}$ center imaging area. The spectral images are produced on the $4 \mathrm{k}$ square center area and moved into the frame stores before readout at high EMCCD gain. During a thruster firing, the sensor is read out as fast as possible to keep the accumulated photon count appreciably below full well.

\subsection{Ultraviolet Channel}

Shown schematically in Figure 9 right, the ultraviolet channel carries a low resolution spectrometer and is also used as the guide channel for IR science. Light from the telescope M3 strikes the fold mirror and then the FSM at the entrance to the starshade instrument. It then reaches a dichroic optic that reflects the UV light to an off-axis paraboloidal mirror and thence through a field stop to an ellipsoidal mirror. Following the ellipse is a filter wheel. Two field stops mounted on a piezoelectric positioning stage are provided, one to allow a field of view up to 10.2 " diameter and another with 0.02" diameter to select individual objects. The beam is then refocused to the focal plane, passing through a filter placed at the intermediate exit pupil which removes light of wavelengths longer than $450 \mathrm{~nm}$. Also at this exit pupil a grism or alternatively, a zero deviation prism can be introduced for low-resolution spectroscopy. With the grism removed, the camera forms an undispersed image. With the introduction of a mirror further downstream, the exit pupil is relayed to the focal plane, forming a pupil image suitable for starshade guiding. The pupil scale need not be large; a $32 \times 32$ pixel image is formed with each pixel covering a $12 \mathrm{~cm}$ square section of the entrance pupil.

The focal plane consists of a single EMCCD (CCD201) operated at $153 \mathrm{~K}$. The chosen type is a modified version of the off-the-shelf item optimized for high UV sensitivity by deep depletion and delta-doping processes (Nikzad et al. 2012), together with a broad band coating to improve response down to $200 \mathrm{~nm}$. Pixel scale is 14 mas. The format is a $1 \mathrm{k} \times 1 \mathrm{k}$ array with adjacent frame store. Again, the CCD will be read out with high gain to minimize read noise and during a thruster firing, the sensor is read out at $1 \mathrm{kHz}$ to keep the accumulated photon count down.

\subsection{Infrared Channel}

The infrared channel is the primary guide channel used for both visible and UV science, and also carries an infrared IFS. When the IFS is being used, guiding is on the UV guide channel. Infrared light entering the instrument passes through both dichroics and is reflected off a paraboloidal mirror (Figure 9 left). Between the second dichroic and the paraboloid, a filter wheel allows band selection. The subsequent layout follows a similar scheme to the UV channel with a focus, ellipsoid and conditioning optics to reach the desired F\#s. At the focus, a fixed field stop limits the field of view to 4", slightly larger than the IFS FOV.

The guide channel consists of a lens to relay the exit pupil following the ellipsoid to the focal plane with the magnification providing a minimum of 32 pixels across the telescope aperture. The focal plane consists of a single linear mode avalanche photodiode (LMAPD) array detector based on an HgCdTe sensor (Saphira array by Selex). The avalanche gain-mode allows the effective read noise to be reduced (but not yet to the extent possible in EMCCDs). The detector is cooled to $60 \mathrm{~K}$ to minimize dark current. Note that an IR imaging mode is easily provided in this layout, though not called for in the STM.

The science channel consists of a powered relay mirror inserted near the guide channel relay lens. This provides the necessary larger focal length to the MLA. Before reaching the MLA, the beam is folded at a plane mirror. A calibration source is provided behind this mirror, injecting through it, so that the position of the spectrum can be identified on the IFS focal plane. The IFS utilizes a planned variant of the Selex detector with a $1 \mathrm{k} \times 1 \mathrm{k}$ format and smaller pixels $(12 \mu \mathrm{m})$ operated at $60 \mathrm{~K}$. Four of these detectors are arrayed in a $2 \times 2$ format to provide a full FOV of $3.8 " \times 3.8 "$ at $\mathrm{R}=40$. The IFS optics follow the same general design as for the visible IFS, with appropriate optical prescription changes. The field of view is Nyquist sampled by the lenslets and likewise, the spectrum is Nyquist sampled at the detector. During a thruster firing, due to its parallel output format and windowing capability, the sensor can be read out at very high frame rates to keep the accumulated charge down and thus avoid contamination of the science data caused by charge persistence. 


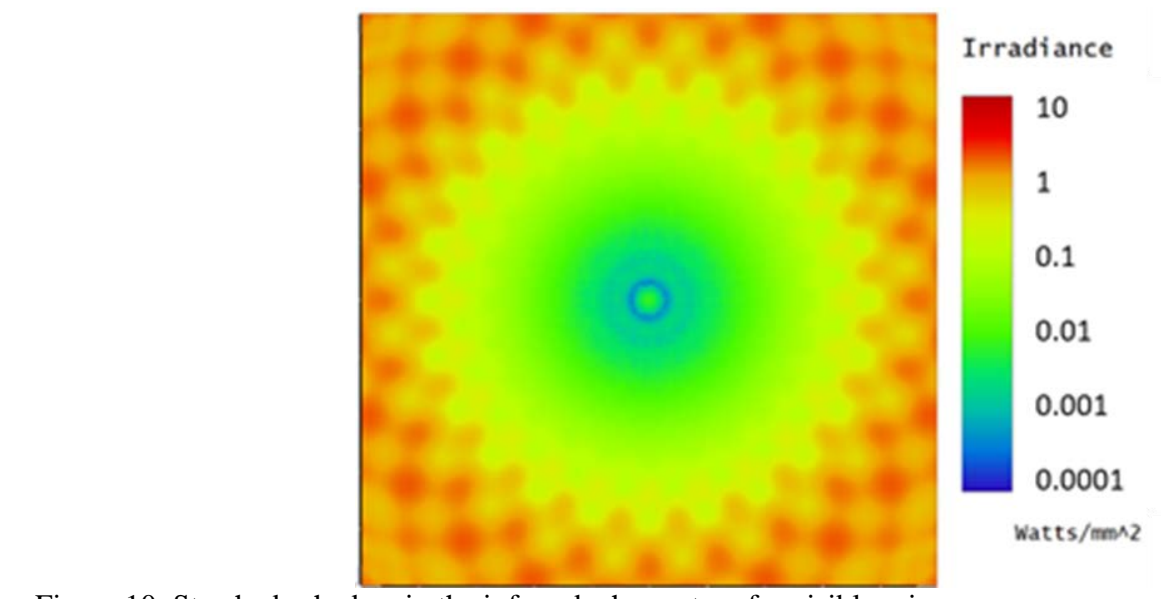

Figure 10: Starshade shadow in the infrared when set up for visible science.

\subsection{Performance and Analysis}

Figure 6 shows the optical transmission of the starshade instrument channels (right) compared to the coronagraph camera channel (left, again with QE and any other effects neglected). Since the starshade instrument requires far fewer mirrors, efficiency is about $67 \%$ better $600 \mathrm{~nm}$ so that spectra and images can be expected to be acquired in a significantly shorter time. Also, the UV channel has excellent performance to $200 \mathrm{~nm}$ and below. The starshade instrument is diffraction limited at the camera focal planes as well as at the input planes of the IFSs.

\subsection{Operations}

Figure 10 shows an image of the starshade shadow structure in infrared light when the starshade is set up for visible science. The central dot appears directly on the line of sight between the center of the starshade and the star and forms the target for the guide system. Outside this core, there are two faint rings and then the flux increases with a monotonic slope towards the edge. Outside this region, a pattern reflective of the starshade geometry appears with, in this case, 24 peaks around a circumference; the HabEx starshade design having 24 petals. The central peak has a diameter approximately equal to $d \lambda D_{\text {ss }}$ where $d$ is the separation distance between the starshade and the telescope, $\lambda$ is the wavelength and $\mathrm{D}_{\mathrm{ss}}$ the starshade diameter. In the case modeled, the central peak is about $3 \mathrm{~m}$ in diameter. The diameter of the smooth, sloping region is approximately $25 \mathrm{~m}$ and a pronounced pattern exists outside this with a diameter of about $50 \mathrm{~m}$. Formation flight and starshade navigation utilize this pattern to bring the starshade into line with the star. Within the patterned region, the starshade follows the gradient down to the center. Once centered, the system maintains the central spot in the telescope pupil by periodically (every $\sim 600 \mathrm{~s}$ ) firing thrusters on the starshade. This alignment is precise because the target stars are bright and the attenuation by the starshade in the guide bands is poor. When a thruster firing occurs, science data taking is briefly suspended $(\sim 1 \mathrm{~s})$. This is because the thruster plumes are illuminated by the Sun and would contaminate the data. However, they rapidly dissipate and the collected flux at the detectors is small; the detectors are electronically cleared and resume data acquisition.

The UV and IR channels have guide camera modes, which project an image of the telescope pupil onto the respective focal planes. With a selected channel in science mode, an optic is introduced into the corresponding guide channel to place an image of the pupil on the guide CCD. The pixel resolution of the starshade's lateral position at the telescope entrance pupil is $12 \mathrm{~cm}$. The lateral position of the starshade is sensed by comparing the image at the entrance pupil to a library of expected images. This lateral sensing/ formation flying technology is at TRL5 $5^{[7]}$. 


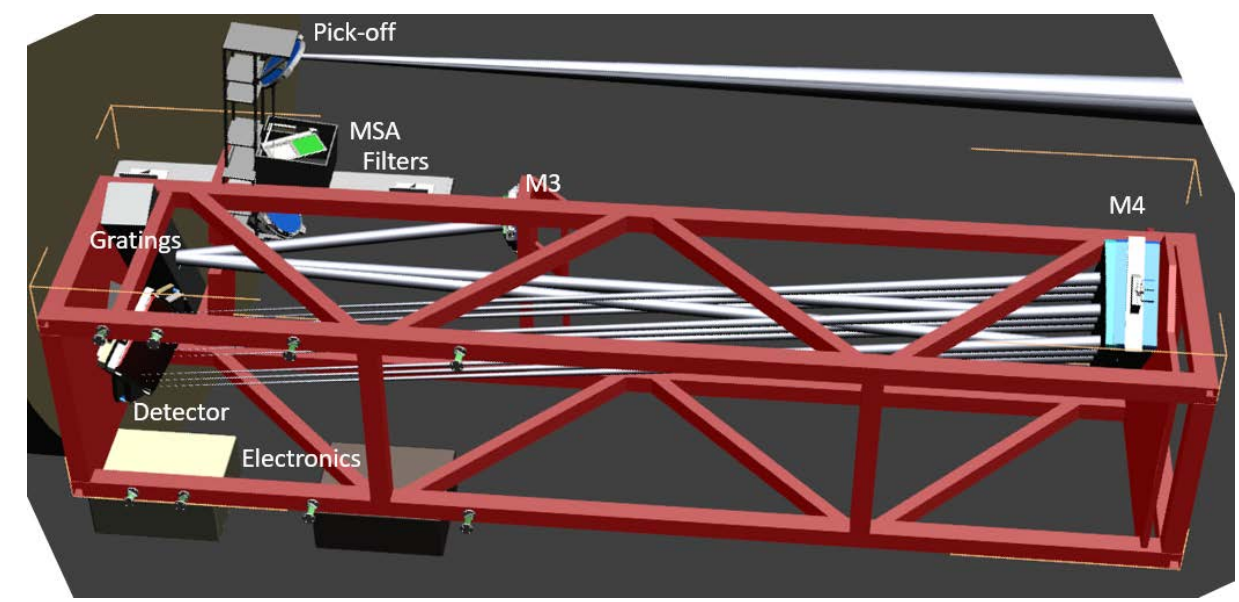

Figure 12: Mechanical view of UVS.

\section{HABEX ULTRAVIOLET SPECTROGRAPH}

\subsection{Design}

The UVS ${ }^{[8]}$ instrument enables high-resolution spectroscopy down to $115 \mathrm{~nm}$ in the UV. The driving science cases include understanding the life cycle of baryonic material as it is moved into and out of galaxies and into stars and planets, determining the escape fraction of hydrogen-ionizing photons from star-forming galaxies and whether this can explain the reionization of the universe at early ages, and the life cycle and impact of massive stars on their environments and the subsequent generations of stars and planets that follow the first generation of massive stars. The needed capabilities include a wide field of view and the ability to perform multi-object spectroscopy (MOS) within that field. The science also calls for access at the shortest wavelengths possible. The baseline design specifies reflectivity down to $115 \mathrm{~nm}$ using $\mathrm{Al}$ coated mirrors protected with MgF2. A range of spectral resolutions is required to enable measurements in both emission and absorption.

The UVS (optical layout shown in Figure 11) utilizes a microshutter array (MSA ${ }^{[9]}$ ) situated at the two-mirror Cassegrain focus to enable selection of numerous individual objects of interest from a $3^{\prime} \times 3$ ' FOV. Table 3 shows key design parameters for the UVS. With a maximum resolution of 60,000, the UVS needs a large set of 20 gratings to cover the wavelength band, plus one plane mirror for imaging. The detector area is large, requiring a $3 \times 5$ array of approximately 100×100 mm microchannel plate (MCP) detectors.

Because of the relatively low reflectance of available mirror coatings in the UV (the reflectivity of aluminum/MgF2 is 61\% @ $115 \mathrm{~nm}$ ), it is important to minimize the number of reflections from the primary mirror to the focal plane. The currently baselined UVS design has 7 reflections in its path compared with 3 and 6 in the narrow field of view COS instrument on Hubble. This design was chosen because of integration conflicts with the other instruments. It contains

Table 3: UVS design parameters. Besides the spectrographic capability, UVS has an imaging mode. In that mode, the microshutter array can be removed from the beam train.

\begin{tabular}{|l|l|}
\multicolumn{1}{|c|}{ Parameter } & \multicolumn{1}{c|}{ Specification } \\
\hline FOV & $3^{\prime} \times 3^{\prime}$ \\
\hline Wavelength bands & 20 bands covering 115 to $320 \mathrm{~nm}$ \\
\hline Spectral resolutions & $\begin{array}{l}60,000 ; 25,000 ; 12,000 ; 6,000 ; \\
3,000 ; 1,000 ; 500\end{array}$ \\
\hline Telescope resolution & Diffraction limited at $400 \mathrm{~nm}$ \\
\hline Detector & $3 \times 5 \mathrm{MCP}$ array, $100 \mathrm{~mm}$ sq each \\
\hline Array width & $17,000 \times 30,000$ pixels (pores) \\
\hline Microshutter & $\begin{array}{l}2 \times 2 \text { array of } 171 \times 365200 \times 100 \mu \mathrm{m} \\
\text { aperture array }\end{array}$ \\
\hline
\end{tabular}



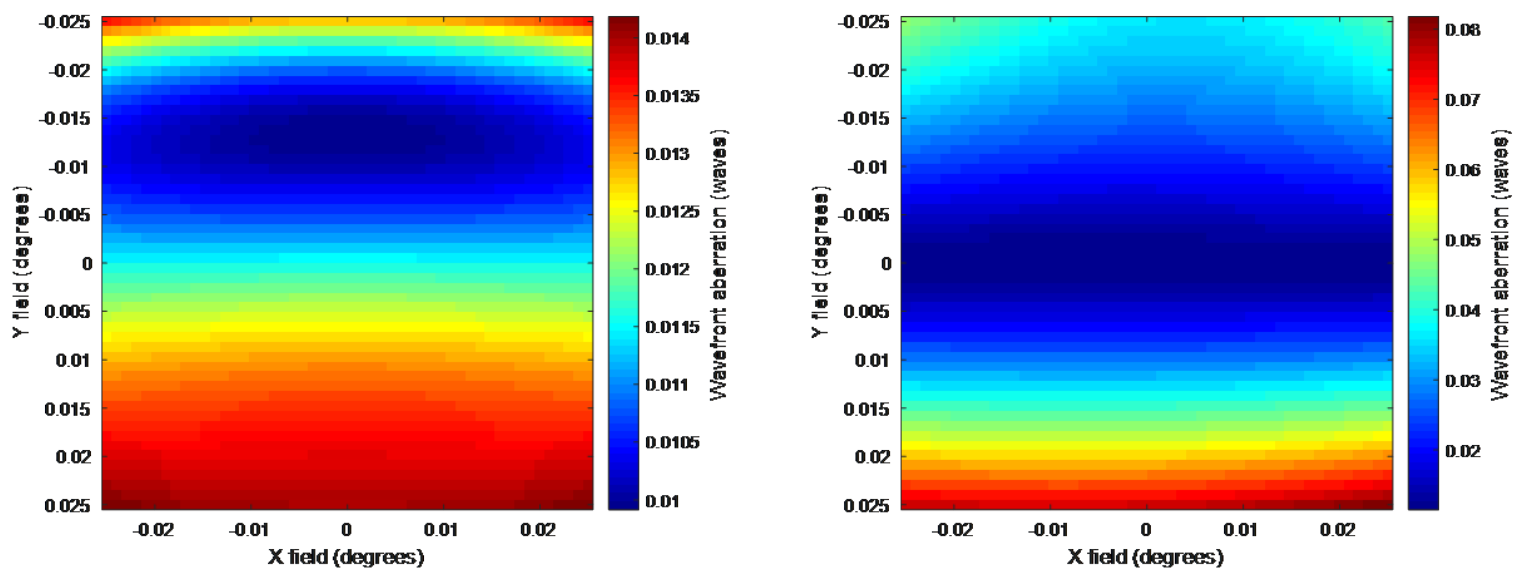

Figure 13: RMS wavefront field maps for UVS: design is diffraction limited (400 nm metric) over the entire field.

two additional folds over the minimum required. In addition, 5 and 6 reflection designs were developed subsequent to the baseline definition and would improve throughput considerably. With a maximum spectral resolution of 60,000 , the dispersion is large, and the focal plane requires about $30,000 \times 17,000$ pixels (or "pores" in the case of microchannel plate detectors) to cover the FOV. In addition to the set of gratings, a set of 7 optical filters is provided to enable rapid spectral observations of particular spectral lines of interest. The set of gratings will be mounted on a cylinder forming four rows of optics. Then by rotating the cylinder about its axis and translating it, any of the gratings (and the one plane mirror used for imaging) can be selected. The optical design is constrained by the telescope's first two mirrors and so a wide design space was explored to reach a solution. The best-corrected focus available is on-axis at the Cassegrain focus formed by the primary and secondary mirrors, so this is where the MSA is located. The beam is folded out of the main telescope path and passes through the MSA and any filter placed in its path. The tertiary mirror is an off-axis aspheric surface, producing an exit pupil at the grating. The grating is plane and carries an evenly spaced groove pattern with approximately $2 \mu \mathrm{m}$ spacing for the shortest wavelength and highest dispersion. This surface can be fabricated using conventional optical polishing plus electron beam lithographic techniques. Following the grating, the beam travels $\sim 3.7$ $\mathrm{m}$ to M4 where it is reimaged onto the detector positioned behind the grating set. The resultant design yields the $3^{\prime} \times 3^{\prime}$ field, corrected at the telescope diffraction limit of $0.4 \mu \mathrm{m}$. Gratings are individually optimized for each waveband and the design includes one optic without grating lines, so that an undispersed UV image is formed.

With a Nyquist sampling criterion for the field of view at $0.4 \mu \mathrm{m}$, the pixel width is equal to $\lambda / 2 \mathrm{~d}$. In the spectral domain, the criterion for spectral elements to be resolved is the same so that a spectral resolution element $\Delta \lambda$ covers two pixels. For example, with $\mathrm{R}=60,000$ at $120 \mathrm{~nm}, \lambda / \Delta \lambda=2 \mathrm{pm}$ and the number of spectral elements needed to cover the first band $12 \mathrm{~nm}$ wide is 6,000. Thus, a single spectrum on the detector will cover 12,000 pixels, resulting in the rectangular shape of the focal plane, longer in the dispersion direction.

The detector is a photon-counting device consisting of an array of glass capillary arrays (GCAs) ${ }^{[10]}$ consisting of thinwalled hexagonal tube assemblies, and are fabricated in very low-Pb glass for a low X-ray cross-section. The tubes (micropores) are arranged with a small angle typically $\sim 15^{\circ}$ off normal. Each plate consists of a micropore array of two layers with opposing pore angles for collection efficiency. Furthermore, the materials used are very pure and contain few radioactive isotopes, leading to a very low dark count. Atomic layer deposition (ALD) is used to create the resistive and emissive layers (GaN and multialkali) of the cathode, producing improved performance over conventional MCPs. Ideally, the assembled plate would be enclosed in a frame maintained under a dry inert gas to assure long-term performance of the emissive layers during integration and testing through launch. However, this concept was considered impractical so MgF2 windows (possible with the large crystal boules now being made) are specified over vacuum sealed detector units. Since the plates are surrounded by the frame of the vacuum assembly there will be small gaps of coverage as is often the case with detector arrays. Beneath the plate a lattice of wires forms the anode. Incoming UV photons produce a cascade of electrons with gain of $10^{6}$ or more, and the charge cloud emerges at the base of the MCP assembly to impact the anode wires. High-speed analog-to-digital converters (ADCs), digitizing 8 bits at $10 \mathrm{MHz}$, collect charge from the wires. An application-specific integrated circuit (ASIC) postprocessor outputs a stream of data consisting of 
charge cloud centroid position (x and y), peak height, coincidence flag, and time stamp. In the case of two or more photons arriving at the same time, the postprocessor rejects the event based on peak height. Behind the anode is a plastic scintillator viewed by a miniature avalanche photodiode or photomultiplier tube that acts as a cosmic ray detector. In the case of a detection here coincident with an event on the wire grid, the coincidence flag is set and the event rejected. Thus, a clean signal can be generated in the presence of a cosmic ray background. The spatial digitization is at the micropore spacing. While the charge cloud spreads upon emission from the base of the plates, the large electron count allows localization of the event at the micropore level. The ADC rate $(10 \mathrm{MHz})$, can handle up to approximately $10^{7}$ photons/sec with a small efficiency loss due to the coincidence of a portion of events. Most targets will be weak, so that typically hundreds of objects may be observed simultaneously.

The optomechanical design is shown in Figure 12. A cage structure supports the system. Most of the components are at one end, the exception being M4. At the base, the MCP array is positioned with its readout electronics. Light is collected by the first fold mirror and passes through the MSA assembly. This assembly can be removed for imaging as it slides on rails, producing an unobstructed field of view. Following this, the filter set is located. Following the second fold (a

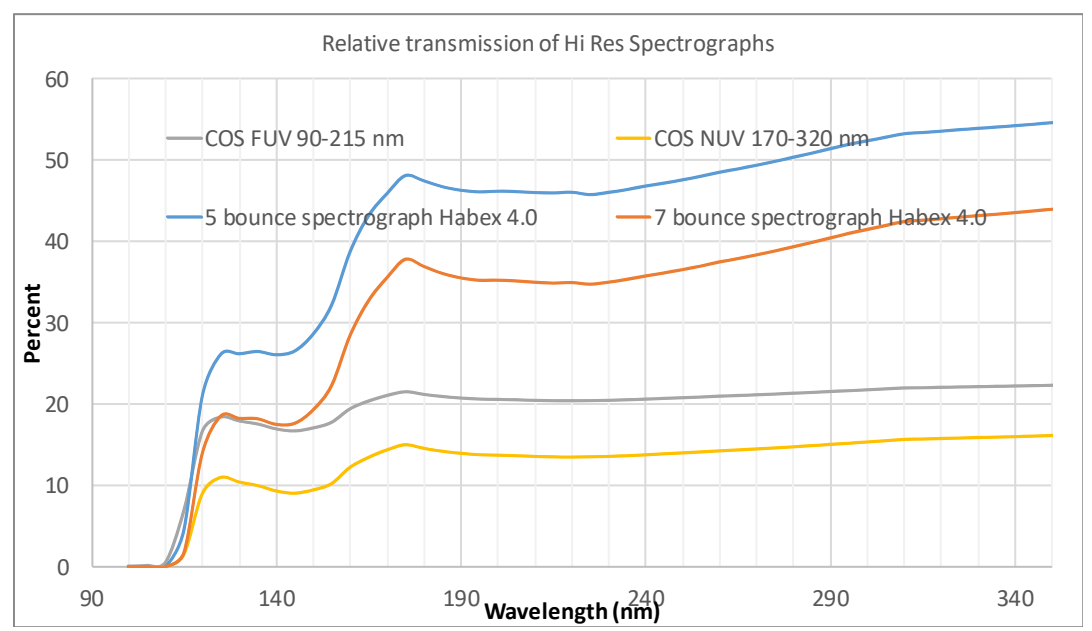

Figure 14: Comparative throughput (relative to the HabEx aperture) of high resolution UV spectrographs.

moveable mirror to correct for focus changes on introduction of a filter) and M3, the gratings are mounted on a cylinder which allows the large number of gratings to be accommodated in a small package. Electronics for the system are mounted on the exterior of the enclosure giving good access to radiators on the telescope exterior.

\subsection{Performance and Analysis}

Figure 13 (left and right) shows the rms spot radius as a function of field for the design for the imaging and high resolution $(\mathrm{R}=60,000)$ modes. The design is diffraction limited across the entire field, measured by the telescope design diffraction wavefront quality at $400 \mathrm{~nm}$. Figure 14 shows the optical throughput of the UVS compared to two Hubble UV channels, considering the relative area of the two telescopes and ignoring any differences in detector performance.

\section{HABEX WORKHORSE CAMERA}

\subsection{Design}

The HabEx workhorse camera (HWC) ${ }^{[11]}$ is a general purpose instrument providing visible through near-IR imaging and spectroscopy, with objectives ranging from solar system science to detailed studies of galaxies and quasars at the epoch of reionization to cosmology. The HWC would enable detailed follow-up of interesting targets, such as those identified from the wide-field surveys of the 2020s, such as Euclid, LSST, and WFIRST. Specifically, the instrument is designed to provide unique scientific capabilities compared to the ground-based facilities expected in the 2030s. For example, nearly all of the first-generation instruments on the new $30 \mathrm{~m}$ class telescopes (e.g., TMT, GMT, and ELT) are near-IR instruments because ground-based adaptive optics (AO) are not expected to be effective for wavelengths much shorter than about $1 \mu \mathrm{m}$. The HWC would provide both unique capabilities, including: (1) high-spatial resolution imaging, (2) a 


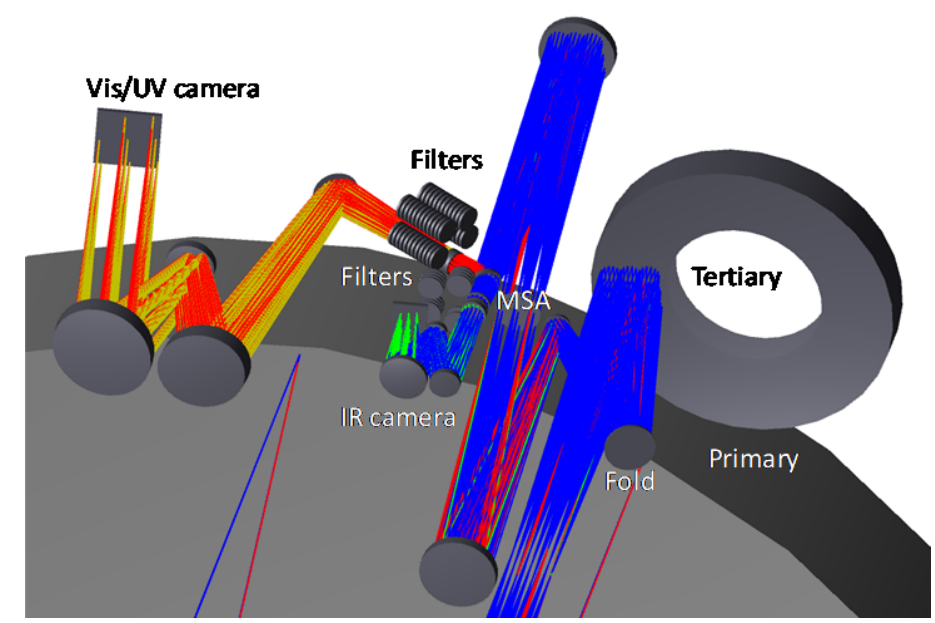

Figure 15: Optical layout of HabEx Workhorse Camera.

stable platform for both photometry and morphology, and (3) access to spectral regions inaccessible on the ground due to telluric absorption.

The design, like the Wide-Field Camera 3 (WFC3) on the HST, has two channels that can simultaneously observe the same field of view: a near UV/optical channel using delta-doped CCD detectors providing good throughput from $370 \mathrm{~nm}$ to $950 \mathrm{~nm}$, and a near-IR channel using Hawaii-4RG HgCdTe arrays providing good throughput from $950 \mathrm{~nm}$ to 1800 $\mathrm{nm}$, at which point thermal backgrounds dominate over most celestial targets.

Both channels will have imaging and spectroscopic modes, and an MSA assembly provides for slit spectroscopy of targeted sources, significantly reducing the backgrounds and source confusion compared to the slit-less spectroscopic modes available on HST. The two modes of operation share the same optical path and cameras. In the spectrographic mode, the MSA and grism sets are introduced into the beam paths. The MSA is attached to a mechanism and thereby removable for the best imaging performance. Table 4 shows the design parameters for the HWC's two channels. For good imaging, the pixel magnification is chosen to Nyquist sample the PSF. To obtain sufficient field of view, the visible channel has a $3 \times 3$ array of $4 \mathrm{k}$ square CCD detectors, and the IR channel utilizes $2 \times 2$ H4RG10.

Figure 15 shows the optical layout of the HWC instrument. After reflecting off M3 and the fold mirror, the input beam strikes a fine-steering mirror used for image dithering and small pointing adjustments and is normally fixed during an observation. The beam then passes through a relay formed by a pair of biconic paraboloidal mirrors, then on to a dichroic where the short wavelength light is separated from the IR light. In spectroscopy mode, a microshutter aperture array is inserted into the focal plane of the relay, enabling selection of particular targets. This MSA array is identical to the set installed in JWST's Near-Infrared Spectrograph (NIRSPEC).

Table 4: Principal design parameters of the HabEx workhorse camera.

\begin{tabular}{|l|c|c|}
\hline FOV & VIS Channel & IR Channel \\
\hline Wavelength bands & $370-975 \mathrm{~nm}$ & $950-1800 \mathrm{~nm}$ \\
\hline Pixel resolution & $15.5 \mathrm{mas}$ & $24.5 \mathrm{mas}$ \\
\hline $\begin{array}{l}\text { Telescope } \\
\text { resolution }\end{array}$ & $30.9 \mathrm{mas}$ & 49 mas \\
\hline Design wavelength & $0.6 \mu \mathrm{m}$ & $0.95 \mu \mathrm{m}$ \\
\hline Detector & $3 \times 3 \mathrm{CCD} 203$ & $2 \times 2$ H4RG10 \\
\hline Detector array width & 12,288 pixels & 8,192 pixels \\
\hline Spectrometer & $\mathrm{R}=1,000$ & $\mathrm{R}=1,000$ \\
\hline Microshutter array & $\begin{array}{l}2 \times 2 \text { arrays; } 180 \times 80 \\
\text { size } ; 171 \times 365 \text { apertures }\end{array}$ \\
\hline
\end{tabular}




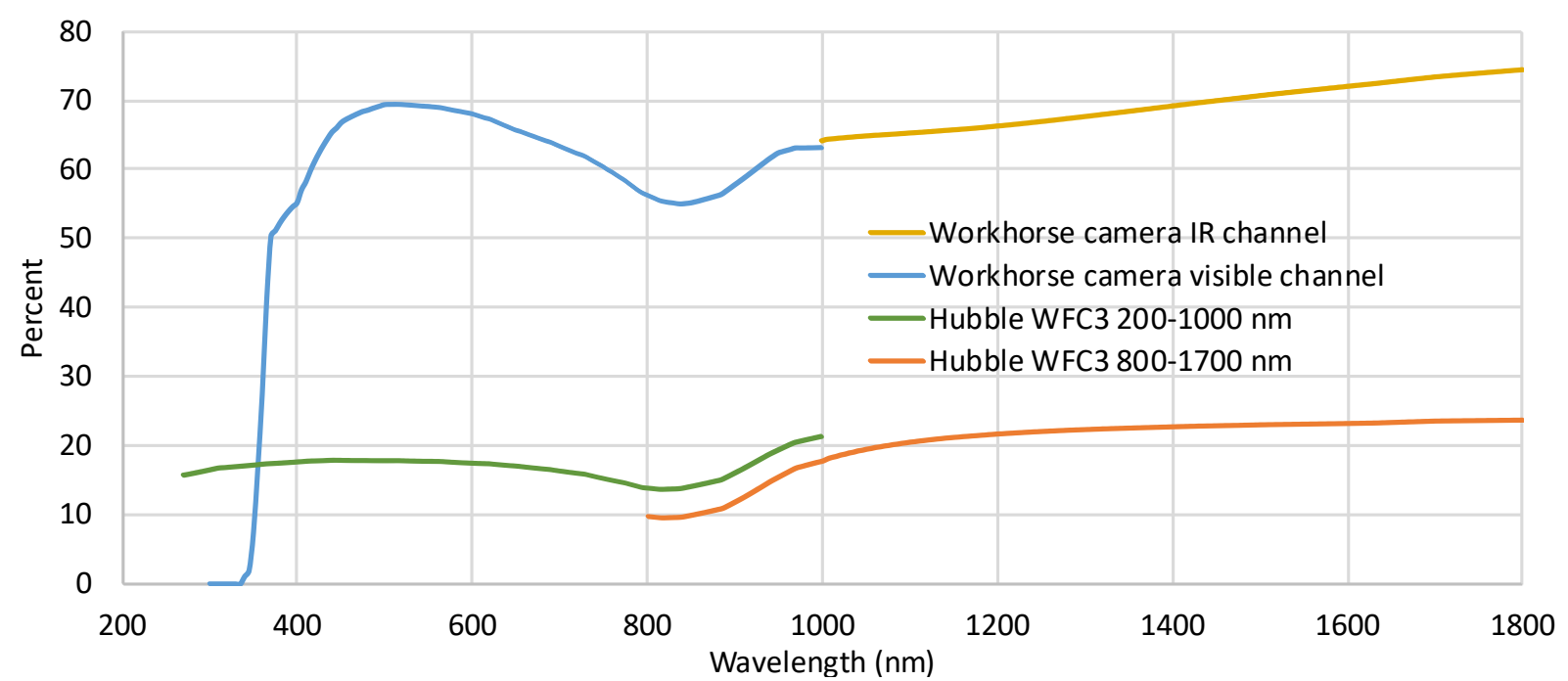

Figure 16: Relative transmission of HWC and Hubble WFC3 channels accounting for aperture area and ignoring other effects. HWC benefits both from a larger telescope aperture and better overall throughput resulting from protected silver coatings.

\subsection{Visible Channel}

After the dichroic, visible light is reflected and passes through a filter wheel to a camera. The filter wheel is mounted at a pupil plane. A grism is also placed in the wheel to allow spectroscopy in conjunction with the MSA at $\mathrm{R}=1,000$. The camera consists of a three-mirror relay and the focal plane itself. The performance is diffraction limited at $400 \mathrm{~nm}$.

The focal plane is designed for Nyquist sampling of the $3^{\prime} \times 3^{\prime}$ field at the same wavelength. The selected array is CCD203, a conventional low-noise CCD with $12 \mu \mathrm{m}$ pixel size and $4 \mathrm{k} \times 4 \mathrm{k}$ format. A set of nine of these deep depletion, delta-doped CCDs, cooled to 153K, forms the focal plane.

\subsection{Infrared Channel}

Infrared light from 950 to $1800 \mathrm{~nm}$ is transmitted through the dichroic and passes via a filter wheel to a camera. As in the visible channel, the filter wheel is mounted at a pupil plane. Again, a grism is placed in the wheel to allow spectroscopy in conjunction with the MSA at $\mathrm{R}=1,000$. The camera consists of a three mirror relay leading to the focal plane. Performance is diffraction limited at $400 \mathrm{~nm}$. The focal plane is designed for Nyquist sampling of the $3^{\prime} \times 3^{\prime}$ field at
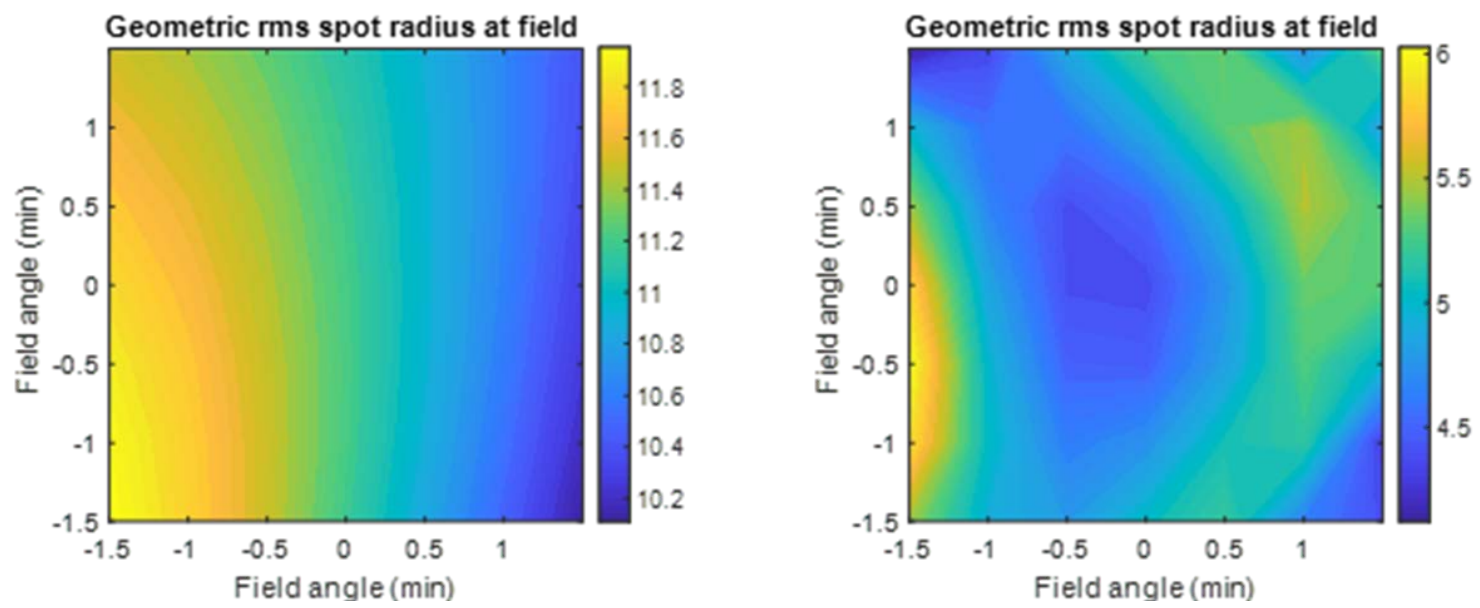

Figure 17: HWC diffraction spot size $(\mu \mathrm{m})$ on the visible and IR channels (left and right respectively): Both focal planes are diffraction limited. 
the $950 \mathrm{~nm}$. The selected array is the Teledyne H4RG10, a low-noise hybrid HgCdTe/CMOS bump-bonded array with $10 \mu \mathrm{m}$ pixel size and $4 \mathrm{k} \times 4 \mathrm{k}$ format. These focal plane arrays (FPAs) are currently being developed for WFIRST. A set of four FPAs cooled to $100 \mathrm{~K}$ forms the focal plane.

\subsection{Performance and Analysis}

Figure 16 shows the optical throughput performance of the visible and infrared channels compared to WFC3, based on aperture area with detector differences excluded. Compared with Hubble's WFC3, HWC has more reflecting surfaces but still posts better throughput by excluding UV operation. In HWC, only two mirror surfaces are $\mathrm{Al}$ and the rest protected silver, which has higher reflectivity above $400 \mathrm{~nm}$ wavelength. Figure 17 shows the diffraction spot sizes across the field; for both the visible and the infrared the cameras are diffraction limited across the whole field of view. Compared with Hubble, HabEx has a larger aperture providing improved angular resolution (1.67× better). The aperture is also unobscured, so the characteristic diffraction from the secondary mirror's spiders seen in Hubble images will not appear.

\section{SUMMARY}

The baseline Habitable Exoplanet Observatory consists of two separate spacecraft flying in formation in an Earth-Sun L2 orbit. One spacecraft carries a $4 \mathrm{~m}$ off-axis telescope and four science instruments - a coronagraph (HCG) and a starshade instrument (SSI) for exoplanet direct imaging, and a wide-field workhorse camera (HWC) and a wide-field high-resolution ultraviolet spectrograph (UVS) for general astronomical observatory science. The other spacecraft carries a $52 \mathrm{~m}$ starshade. With the starshade flying in formation on axis with the telescope, an external occulter observatory for exoplanet imaging and spectral characterization is formed. The instrument payload is designed not only to deliver ground-breaking exoplanet science, but to supersede the performance of the Hubble Space Telescope and provide a next generation Great Observatory to follow on from WFIRST and JWST.

\section{ACKNOWLEDGEMENTS}

The information in this paper is provided prior to any decisions or commitments being made and is provided for planning and discussion purposes only. This work was conducted at the Jet Propulsion Laboratory, California Institute of Technology, under contract with the National Aeronautics and Space Administration. Copyright 2019 California Institute of Technology. Government sponsorship acknowledged. All rights reserved.

\section{REFERENCES}

[1] H. Philip Stahl, “Habitable exoplanet imager optical telescope concept design”, Proc. SPIE. 10398, 5 September 2017.

[2] Stefan Martin, Mayer Rud, Paul Scowen, Daniel Stern, Joel Nissen, John Krist, "HabEx space telescope optical system”, Proc. SPIE. 10398, 5 September 2017

[3] Fang Shi, Byoung-Joon Seo, Eric Cady, Brian Kern, Raymond Lam, David Marx, Keith Patterson, Camilo Mejia Prada, John Shaw, Chris Shelton, Joel Shields, Hong Tang, Tuan Truong, "WFIRST low order wavefront sensing and control dynamic testbed performance under the flight like photon flux," Proc. SPIE 10698, Space Telescopes and Instrumentation 2018: Optical, Infrared, and Millimeter Wave, $106982 \mathrm{O}$ (30 July 2018)

[4] Michael W. McElwain et al. "PISCES: an integral field spectrograph technology demonstration for the WFIRST coronagraph,” Proc. SPIE 9904, (29 July 2016).

[5] Jonas Kühn, Sebastian Daemgen, Ji Wang, Farisa Morales, Michael Bottom, Eugene Serabyn, Jean C. Shelton, Jacques-Robert Delorme, Samaporn Tinyanont, "High-contrast imaging of tight resolved binaries with two vector vortex coronagraphs in cascade with the Palomar SDC instrument," Proc. SPIE 10702, Ground-based and Airborne Instrumentation for Astronomy VII, 1070242 (27 July 2018);

[6] N. J. Kasdin, D. N. Spergel, R. J. Vanderbei, D. Lisman, S. Shaklan, M. Thomson, P. Walkemeyer, V. Bach, E. Oakes, E. Cady, S. Martin, L. Marchen, B. Macintosh, R. E. Rudd, J. Mikula, D. Lynch, "Advancing technology for starlight suppression via an external occulter," Proc. SPIE 8151, Techniques and Instrumentation for Detection of Exoplanets V, 81510J (15 September 2011) 
[7] Flinois et al., "S5 Milestone 4: Lateral formation sensing and control”, https://exoplanets.nasa.gov/internal_resources/1077.

[8] Paul A. Scowen, Stefan Martin, Mayer Rud, Gary Kuan, Daniel Stern, Alina Kiessling, Bertrand Mennesson, Keith Warfield, "HabEx ultraviolet spectrograph design and DRM," Proc. SPIE 10699, Space Telescopes and Instrumentation 2018: Ultraviolet to Gamma Ray, 1069905 (6 July 2018);

[9] Mary Li, Tomoko Adachi, Christine Allen, Sachi Babu, Sateesh Bajikar, Michael Beamesderfer, Ruth Bradley, Kevin Denis, Nick Costen, Audrey Ewin, David Franz, Larry Hess, Ron Hu, Kamili Jackson, Murzy Jhabvala, Dan Kelly, Todd King, Gunther Kletetschka, Alexander Kutyrev, Barney Lynch, Timothy Miller, Harvey Moseley, Vilem Mikula, Brent Mott, Lance Oh, James Pontius, David Rapchun, Chris Ray, Eric Schulte, Scott Schwinger, Peter Shu, Robert Silverberg, Wayne Smith, Steve Snodgrass, David Sohl, Leroy Sparr, Rosalind Steptoe-Jackson, Valeriano Veronica, Liqin Wang, Yun Zheng, Chris Zincke, "MEMS microshutter arrays for James Webb Space Telescope”, Proc. SPIE. 6415, 20 December 2006

[10] C. D. Ertley, O. H. W. Siegmund, S. R. Jelinsky, J. Tedesco, M. J. Minot, A. O’Mahony, C. A. Craven, M. Popecki, A. V. Lyashenko, M. R. Foley, "Second generation large area microchannel plate flat panel phototubes," Proc. SPIE 9915, High Energy, Optical, and Infrared Detectors for Astronomy VII, 99152G (27 July 2016);

[11]Daniel Stern, John Clarke, Scott Gaudi, Alina Kiessling, Oliver Krause, Stefan Martin, Paul Scowen, Rachel Somerville, "The HabEx workhorse camera (Conference Presentation)," Proc. SPIE 10698, Space Telescopes and Instrumentation 2018: Optical, Infrared, and Millimeter Wave, 106980R (10 July 2018) 